\title{
Holomorphic non-holonomic differential systems on complex manifolds
}

\author{
by S. Dimiev (Sofia)
}

\begin{abstract}
We study coherent subsheaves $\mathcal{D}$ of the holomorphic tangent sheaf of a complex manifold. A description of the corresponding $\mathcal{D}$-stable ideals and their closed complex subspaces is sketched. Our study of non-holonomicity is based on the Noetherian property of coherent analytic sheaves. This is inspired by the paper [3] which is related with some problems of mechanics.
\end{abstract}

\section{Systems of holomorphic vector fields and integral subspaces.} Let $M$ be a complex $\nu$-manifold $\left(\operatorname{dim}_{\mathbb{C}} M=\nu\right)$. Let $\mathcal{O}_{M}$ be the structure sheaf of $M$ and let $\mathcal{T}_{M}$ be the holomorphic tangent sheaf of $M\left(\mathcal{T}_{M}=\right.$ $\left.\operatorname{Der}_{\mathbb{C}} \mathcal{O}_{M}\right)$. We say that each submodule $\mathcal{D}$ of $\mathcal{T}_{M}$ of finite type defines a holomorphic differential system of first order or a system of holomorphic vector fields on $M$. In fact, $\mathcal{D}$ is a coherent $\left(\mathcal{O}_{M}\right.$-coherent) subsheaf of $\mathcal{T}_{M}$, as $\mathcal{T}_{M}$ is a locally free sheaf. The local sections of $\mathcal{D}$ are differential operators of first order with holomorphic coefficients, i.e. holomorphic vector fields. For each open subset $U$ of $M, \mathcal{D}(U)$ is an $\mathcal{O}_{M}$-module, i.e. if $\Delta \in \mathcal{D}(U)$ and $f \in \mathcal{O}_{M}(U)$ then $f \Delta \in \mathcal{D}(U)$ etc.

We denote by $\mathcal{L D}$ the minimal Lie algebra subsheaf of $\mathcal{T}_{M}$ which contains $\mathcal{D}$, i.e. $\mathcal{D} \subset \mathcal{L D} \subset \mathcal{T}_{M}$. This means that for every $p \in M$ the stalk $\mathcal{D}_{p}$ is contained in the stalk $(\mathcal{L D})_{p}$ and the following condition is satisfied: if $\mathfrak{J}$ is a Lie algebra subsheaf of $\mathcal{T}_{M}$ such that $\mathcal{D}_{p} \subset \mathfrak{J}_{p}$ for each $p \in M$, then $(\mathcal{L D})_{p} \subset \mathfrak{J}_{p}$ for each $p \in M$.

Let $G$ be a subset of $M$. We say that the differential system $\mathcal{D}$ is holonomic on $G$ iff $\mathcal{D}|G=\mathcal{L} \mathcal{D}| G$. In the case $\mathcal{D}|G \neq \mathcal{L} D| G \varsubsetneqq \mathcal{T}_{M} \mid G$, we say that $\mathcal{D}$ is a non-holonomic differential system. In the case $\mathcal{D}|G \neq \mathcal{L} \mathcal{D}| G=\mathcal{T}_{M} \mid G$, we say that $\mathcal{D}$ is completely non-holonomic. Recall that $\mathcal{D} \mid G$ denotes the restriction of $\mathcal{D}$ on $G$.

1991 Mathematics Subject Classification: Primary 32B99, 32L05.

Key words and phrases: holomorphic tangent sheaf, $\mathcal{D}$-stable ideal, power $\mathcal{D}$-expansion, involutive completion. 
We also recall that a complex space $X$ is called a closed complex subspace of $M$ if there is a coherent ideal $I$ of $\mathcal{O}_{M}, I \subset \mathcal{O}_{M}$, such that $X=\operatorname{supp}\left(\mathcal{O}_{M} / I\right)$ and $\mathcal{O}_{x}=\left(\mathcal{O}_{M} / I\right) \mid X$. In this case there is a canonical holomorphic map determined by the injection and denoted by $X \subset M$. The tangent space of $X$, denoted by $T X$, is defined as usual [1]. If $G$ is an open subset of $M$ and $\mathcal{O}_{G}$ is the induced structure sheaf, we assume that the ideal $I$ is generated on $G$ by $f_{1}, \ldots, f_{\nu} \in \mathcal{O}_{G}(G)$. If $\left(z_{1}, \ldots, z_{\nu}, s_{1}, \ldots, s_{\nu}\right)$ are coordinates on $G \times \mathbb{C}^{\nu}$, then $T X \subset G \times \mathbb{C}^{\nu}$ is defined as the closed subspace generated by $f_{1}, \ldots, f_{\nu},\left(\partial f_{k} / \partial z_{1}\right) s_{1}, \ldots,\left(\partial f_{k} / \partial z_{\nu}\right) s_{\nu}, k=1, \ldots, \nu$, where $f_{k}$ and $\partial f_{k} / \partial z_{j}$ are viewed as holomorphic functions on $G \times \mathbb{C}^{\nu}$ via the canonical projection $G \times \mathbb{C}^{\nu} \rightarrow G$.

We say that $X$ is an integral subspace for $\mathcal{D}$ or a singular integral of $\mathcal{D}$ if each vector field $\Delta \in \mathcal{D}$ admits a restriction to a vector field on $X$, i.e. to a vector field of the type $X \rightarrow T X$. The following proposition is well known.

Proposition 1.1. The closed complex subspace $X$ defined by $I$ is an integral subspace for the differential system $\mathcal{D}$ iff the ideal I is stable relative to $\mathcal{D}$, i.e. $\mathcal{D}(I) \subset I$, which means that $\Delta(I) \subset I$ for every vector field $\Delta \in \mathcal{D}$.

Such an ideal will be called a $\mathcal{D}$-stable ideal.

2. Involutive completion. If $\mathcal{A}_{U}$ and $\mathcal{B}_{U}$ are submodules of $\mathcal{T}_{M}, U$ being an open subset of $M$, we denote by $\left[\mathcal{A}_{U}, \mathcal{B}_{U}\right]$ the submodule of $\mathcal{T}_{M}(U)$ generated by all vector fields $\Delta \in \mathcal{A}_{U}, \Delta^{\prime} \in \mathcal{B}_{U}$ and all brackets $\left[\Delta, \Delta^{\prime}\right]$.

We shall consider the following increasing sequence of submodules of $\mathcal{T}_{M}(U)$

$$
\begin{aligned}
& \mathcal{D}_{1}(U):=\mathcal{D}(U), \quad \mathcal{D}_{2}(U):=\left[\mathcal{D}_{1}(U), \mathcal{D}_{1}(U)\right], \ldots, \\
& \mathcal{D}_{j}(U):=\left[\mathcal{D}_{j-1}(U), \mathcal{D}_{1}(U)\right], \ldots
\end{aligned}
$$

For each $j \in \mathbb{N}$ the presheaf $\mathcal{D}_{j}=\left\{\mathcal{D}_{j}(U), \rho_{V}^{U}\right\}$ (where $\rho_{V}^{U}$ is as usual the restriction operator from $U$ to $V, V \subset U$ ) is a (canonical) sheaf, which is a subsheaf of $\mathcal{T}_{M}$.

Since by assumption $\mathcal{D}_{1}$ is of finite type, the same is true for $\mathcal{D}_{2}$. One proves by induction that for each $j \in \mathbb{N}$ the sheaf $\mathcal{D}_{j}$ is of finite type. It follows that $\mathcal{D}_{j}$ is also an $\mathcal{O}_{M}$-coherent subsheaf of $\mathcal{T}_{M}$.

Proposition 2.2. Every increasing sequence of coherent sheaves $\left\{\mathcal{D}_{j}\right\}$ on a complex space $Y$ is stationary over any relatively compact subset of $Y$.

The proof is by induction (see [2]). The proposition holds for empty spaces (of dimension less than 0). Assume it is true for all complex spaces of dimension less than $\mu \geq 0$. As $\operatorname{dim}_{y} Y \leq \mu$ is equivalent to there being an open neighborhood $U$ of $y$ and a finite holomorphic map $f: U \rightarrow D$, where $D$ is a connected open set in $\mathbb{C}^{\mu}$, by using the reduction steps of [2] 
(Ch. 5) it is enough to verify the proposition for the structure sheaf $\mathcal{O}_{Y}$ and for connected domains $D$ in $\mathbb{C}^{\mu}$, i.e. for $\mathcal{O}_{D}$. Finally, we use the fact that closed complex subspaces of $D$ are nowhere dense in $D$, which implies that their dimension is strictly less than $\mu$. Indeed, in this case all sheaves $\mathcal{D}_{j}$ are coherent ideals. Let $\mathcal{D}_{j_{0}} \neq 0$. The complex space $Y_{j_{0}}$ of $D$ defined by the ideal $\mathcal{D}_{j_{0}}$ is different from $D$ and according to the above remark we have $\operatorname{dim} Y_{j_{0}}<\mu$. Taking the sequence of all ideals $\mathcal{D}_{j}$ such that $\mathcal{D}_{j} \supset \mathcal{D}_{j_{0}}$ we conclude by the induction hypothesis that the family $\left\{\mathcal{D}_{j}\right\}$ is stationary over any relatively compact subset of $Y$. Of course, we have in mind that all ideals $\mathcal{D}_{j}$ with $\mathcal{D}_{j} \supset \mathcal{D}_{j_{0}}$ are coherent over $Y_{j_{0}}$ in a natural way. The proof is finished.

So, for a compact subset $K$ of $M$ there exist integers $j$ such that for every $p \in K, \mathcal{D}_{j}(p)=\mathcal{D}_{j+1}(p)=\ldots$ The minimal such $j$ will be denoted by $h(K)$. In the case $h(K)=1$, the system $\mathcal{D}$ is holonomic (or involutive) on $K$. If $h(K)=1$ for all compact subsets of $M$, the system is holonomic on $M$ in the usual sense. If $h(K)>1$, the system $\mathcal{D}$ is non-holonomic on $K$. The integer $h(K)$ is called the index of non-holonomicity on $K$. In the case $(\mathcal{L D})_{p}=\left(\mathcal{T}_{M}\right)_{p}$ for every $p \in K$, the system $\mathcal{D}$ is completely non-holonomic on $K$.

Proposition 2.3. Let $U$ be an open connected domain in $M$ and let $\left\{\mathcal{D}_{j}\right\}$ be the sequence (2.1), which is by assumption non-holonomic on $U$. Then the subset $\mathcal{Q}_{n}(U, \mathcal{D})=\{p \in U: h(\{p\})=n\}$, where $n$ is a positive integer, is an analytic subset of $U$.

Pr o o f. Denoting by $\left\{\Delta_{1}, \ldots, \Delta_{k}\right\}=B_{1}$ the base of $\mathcal{D}_{1}(U)=\mathcal{D}(U)$, we consider the following base $B_{2}$ for $\mathcal{D}_{1}(U)$ :

$$
B_{2}=\left\{\Delta_{1}, \ldots, \Delta_{k},\left[\Delta_{j_{1}}, \Delta_{j_{2}}\right]: j_{1}, j_{2}=1, \ldots, k\right\}
$$

(the order of the vector fields included in $B_{2}$ is fixed), etc. The base $B_{l}$ is defined by induction:

$$
B_{l}=\left\{\Delta_{1}, \ldots, \Delta_{k},\left[\Delta_{j_{1}}, \Delta_{j_{2}}\right],\left[\left[\Delta_{j_{1}}, \Delta_{j_{2}}\right], \Delta_{j_{3}}\right], \ldots\right\}, \quad l \in \mathbb{N} .
$$

In such a way we obtain an increasing sequence of bases $\left\{B_{l}\right\}$.

Now, the condition that $h(\{p\})=n$ can be formulated by means of the last member of the base $B_{n+1}$. In fact, $B_{n+1}=B_{n}$ implies the equality

$$
\left.\left[\ldots\left[\left[\Delta_{j_{1}}, \Delta_{j_{2}}\right], \Delta_{j_{3}}\right] \ldots \Delta_{j_{n+1}}\right] \ldots\right]=\sum_{j_{1}, \ldots, j_{n}} C_{j_{1} \ldots j_{n}} \Delta_{j_{1} \ldots j_{n}}
$$

where $\Delta_{j_{1} \ldots j_{n}} \in B_{n}$. Having in mind that (2.4) is satisfied for every $f \in$ $\mathcal{O}(U)$, and calculating the explicit coordinate representation of all relevant vector fields, we conclude that the coefficients on the right and left side are zero at $p$. But they are holomorphic functions on $U$ and this zero-set is an analytic set in $U$. 
3. Power $\mathcal{D}$-expansions. Locally we shall work with power $\mathcal{D}$-expansions. For this purpose we can assume that $U$ is an open neighborhood of the origin $O$ in $\mathbb{C}^{\nu}$ with coordinates $\left(z_{1}, \ldots, z_{\nu}\right)$. As in the previous paragraph, $\Delta_{1}, \ldots, \Delta_{k}$ are holomorphic vector fields on $U$ which are generators for the $\mathcal{O}_{M}$-module $\mathcal{D}(U)$. The notion of power $\mathcal{D}$-expansion or power $\mathcal{D}$-series is based on the coordinate representation of the generators $\Delta_{j}$ :

$$
\Delta_{j}=\sum_{i=1}^{\nu} \Delta_{j}^{i}(z) \frac{\partial}{\partial z_{i}}, \quad\left(z_{i}\right)=z \in U, \Delta_{j}^{i}(z) \in \mathcal{O}(U) .
$$

For a given multi-index $\alpha=\left(\alpha_{1}, \ldots, \alpha_{k}\right)$ we denote by $\Delta^{\alpha}$ the composition

$$
\Delta^{\alpha}:=\Delta_{1}^{\alpha_{1}} \ldots \Delta_{k}^{\alpha_{k}} .
$$

In the case $\Delta_{j}=\partial / \partial z_{j}$ we write $D^{\alpha}$ instead of $\Delta^{\alpha}$.

We assume in the sequel that the vector fields $\Delta_{j}$ appear in a fixed order in the sequence $\Delta_{1}, \ldots, \Delta_{k}$.

In the case when $U$ is a polydisc in $\mathbb{C}^{\nu}$ with center at the origin $O$ and equal radii $\left(r_{1}=\ldots=r_{\nu}=r\right)$ we have the Cauchy inequality

$$
\left|D^{\alpha} g(0)\right| \leq C \alpha ! / r^{|\alpha|}
$$

for every holomorphic function $g$ on $U$.

LEMMA 3.4 (Cauchy inequality for $\Delta^{\alpha} g$ ). Under the above assumptions we have

$$
\left|\left(\Delta^{\alpha} g\right)(0)\right| \leq C^{|\alpha|+1} \nu^{|\alpha|}(|\alpha| !)^{2} / r^{|\alpha|}
$$

Proof. It is not difficult to prove by induction on the length of the multi-index $\alpha$ that $\Delta^{\alpha}(f)$ contains $|\alpha| ! \nu^{|\alpha|}$ summands of the type

$$
\Delta_{i_{0}}^{j_{0}}(z)\left(D^{\beta^{1}} \Delta_{i_{1}}^{j_{1}}(z)\right) \ldots\left(D^{\beta^{n-1}} \Delta_{i_{n-1}}^{j_{n-1}}(z)\right) D^{\beta^{n}} f(z) .
$$

where $n:=|\alpha|$ and $\beta^{1}, \ldots, \beta^{n}$ is a multi-index such that $\left|\beta^{1}\right|+\ldots+\left|\beta^{n}\right|=|\alpha|$. From (3.3) it follows that

$$
\left|\Delta_{i_{0}}^{j_{0}}(0) D^{\beta^{1}} \Delta_{i_{1}}^{j_{1}}(0) \ldots D^{\beta^{n-1}} \Delta_{i_{n-1}}^{j_{n-1}}(0) D^{\beta^{n}} f(0)\right| \leq C^{n+1}|\alpha| ! / r^{|\alpha|},
$$

where $C$ is the common constant in (3.3) for every pair $(i, j), j=1, \ldots, k$ and $i=1, \ldots, n$, i.e. for every $g=\Delta_{j}^{i}$.

Combining the above remark on the number of summands of $\Delta^{\alpha}(f)$ with the last inequality we obtain (3.4).

In the sequel we also need the inequality

$$
|\alpha| ! / \alpha ! \leq C_{1}|\alpha| \nu^{|\alpha|},
$$

where $\alpha !=\alpha_{1} ! \ldots \alpha_{k}$ !, which can be proved with the help of the Stirling formula. 
Having a differential system $\mathcal{D}$ on $U$, we consider the formal power series

$$
T_{\mathcal{D}}(f):=\sum_{\alpha} \frac{\Delta^{\alpha}(f)(0)}{|\alpha| ! \nu^{|\alpha|} \alpha !} z^{2 \alpha},
$$

where $2 \alpha$ is the multi-index $\left(2 \alpha_{1}, \ldots, 2 \alpha_{\nu}\right)$. On the polydisc with common radius $r$ (i.e. for $\left.\left|z_{j}\right|<r, j=1, \ldots, \nu\right)$ we have $|2 \alpha|=2|\alpha|$, etc.

In the classical case of the Frobenius system $\left(\partial / \partial z_{1}, \ldots, \partial / \partial z_{\nu}\right)$ the following remark holds. Since the ordinary Taylor expansion of $f$ about the origin is

$$
\sum_{\alpha} \frac{D^{\alpha}(f)(0)}{\alpha !} z^{\alpha}
$$

we see that in the case of convergent series, $T_{\mathcal{D}}(f)$ converges faster than the ordinary Taylor expansion. In fact, in this case we have

$$
T_{\mathcal{D}}(f):=\sum_{\alpha} \frac{D^{\alpha}(f)(0)}{\alpha !} z^{2 \alpha} .
$$

LEMMA 3.8 (Convergence lemma). The formal power series (3.7) is convergent near the origin, i.e. on polydiscs with common radius $r$ sufficiently small.

Proof. The series (3.7) can be represented as an expansion into homogeneous polynomials

$$
\sum_{n}\left(\sum_{|\alpha|=n} \frac{\Delta^{\alpha}(f)(0)}{|\alpha| ! \nu|\alpha| \alpha !} z^{2 \alpha}\right)
$$

First, we give an estimate for each homogeneous member. Having in mind that if $\left|A_{\alpha}\right| \leq A$ then $\left|\sum_{|\alpha|=n} A_{\alpha}\right| \leq n^{\nu} A$ (recall that $\nu$ is the number of components of the multi-index $\alpha$ ) we get

$$
\left|\sum_{|\alpha|=n} \frac{\Delta^{\alpha}(f)(0)}{|\alpha| ! \nu^{|\alpha|} \alpha !} z^{2 \alpha}\right| \leq n^{\nu} \frac{C^{|\alpha|+1}|\alpha| !}{\alpha !} r^{|\alpha|} \leq C C_{1} n^{\nu+1}(C \nu r)^{n}
$$

in view of (3.6).

Finally, the series (3.7) is convergent on the mentioned polydiscs with $r<1 /(C \nu)$.

4. Construction of $\mathcal{D}$-stable ideals. According to (1.1) the integral subspaces of the holomorphic differential system $(M, \mathcal{D})$ are defined by $\mathcal{D}$-stable ideals of $\mathcal{O}_{M}$. Denote by $\left(f_{r+1}, \ldots, f_{\nu}\right)$ the ideal generated by $\nu-r$ holomorphic functions $f_{j}$ defined on a neighborhood $U$ of the origin in $\mathbb{C}^{\nu}$. We suppose that this ideal defines a germ of integral subspace which passes 
through the origin $\left(f_{r+1}(0)=\ldots=f_{\nu}(0)=0\right)$. Let $J_{\mathcal{D}}$ be the ideal of all $f \in \mathcal{O}_{M}(U)$ such that $\Delta^{\alpha}(f)(0)=0$ for all multi-indices $\alpha$.

Proposition 4.1. Every $\mathcal{D}$-stable ideal $\left(f_{r+1}, \ldots, f_{\nu}\right)$ is contained in the ideal $J_{\mathcal{D}}$.

Proof. If $\left(f_{r+1}, \ldots, f_{\nu}\right)$ is $\mathcal{D}$-stable, then for every $f \in\left(f_{r+1}, \ldots, f_{\nu}\right)$ we have $\Delta^{\alpha}(f) \in\left(f_{r+1}, \ldots, f_{\nu}\right)$, which implies that $\Delta^{\alpha}(f)(0)=0$ for all multi-indices $\alpha$.

In the sequel we need the notion of embedding dimension of a closed complex subspace $X$, and also the well known Jacobi Criterion. For every $p \in X$ there exists a smallest positive integer, denoted by $\operatorname{emb}_{p} X$, such that a neighborhood $V$ of $p$ is holomorphic to a closed complex subspace of a domain in $\mathbb{C}^{\mathrm{emb}_{p} X}$.

LEMma 4.2 (Jacobi criterion). Let $X$ be a closed subspace of a domain $D \in \mathbb{C}^{v}$. If $p \in X$ and $f_{1}, \ldots, f_{l} \in \mathcal{O}(D)$ are such that

$$
\mathcal{O}_{X, p}=\mathcal{O}_{D, p} /\left(f_{1 p}, \ldots, f_{l p}\right) \mathcal{O}_{D, p}
$$

then

(Here $f_{j p}, j=1, \ldots, l$, denote the germs of $f_{j}$ at $p$.)

The proof is based on the implicit function theorem.

In general, $\operatorname{dim}_{p} X \leq \operatorname{emb}_{p} X$ for all $p \in X$. The following proposition is also well known.

Lemma 4.3 (Criterion of smoothness). A point $p \in X$ is smooth iff $\operatorname{emb}_{p} X=\operatorname{dim}_{p} X$.

Recall that $p \in X$ is smooth if there exists a neighborhood of $p$ in $X$ which is biholomorphic to an open neighborhood in $\mathbb{C}^{\mu}$ for some $\mu$.

PROPOSITION 4.4. If the rank at the origin of the (globally non-holonomic) differential system $(M, \mathcal{D})$ is $r$, then

1) there exist $\nu-r$ convergent power $\mathcal{D}$-expansions $g_{j}\left(z_{1}, \ldots, z_{r}\right), j=$ $r+1, \ldots, \nu$, such that the ideal generated by $w_{j}-g_{j}\left(z_{1}, \ldots, z_{r}\right), j=r+$ $1, \ldots, \nu$, is $\mathcal{D}$-stable only if

$$
\Delta^{\alpha}\left(g_{j}\right)(0)=\Delta^{\alpha}\left(z_{j}\right)(0), \quad j=r+1, \ldots, \nu,
$$

for all multi-indices $\alpha$,

2) the closed complex subspace defined by the above ideal $\left(w_{j}-\right.$ $\left.g_{j}\left(z_{1}, \ldots, z_{r}\right)\right)$ is a complex manifold.

Proof. As the dimension of the stalk $\mathcal{D}(0)$ of $\mathcal{D}$ does not depend on the chosen coordinates $\left(z_{1}, \ldots z_{r}, w_{r+1}, \ldots, w_{\nu}\right)$, we can suppose that after 
some renumbering, the vectors $\left(\Delta_{1}(0), \ldots, \Delta_{r}(0)\right)$ form a base for $\mathcal{D}(0)$. This means that the matrix

$$
\left\|\Delta_{j}^{i}(0)\right\|, \quad i, j=1, \ldots, r,
$$

is non-singular. By means of a suitable change of coordinates the following canonical form for the generators $\Delta_{j}$ can be obtained:

$$
\begin{aligned}
& \Delta_{1}=\frac{\partial}{\partial z_{1}}+\Delta_{r+1}^{1} \frac{\partial}{\partial z_{r+1}}+\Delta_{\nu}^{1} \frac{\partial}{\partial z_{\nu}}, \\
& \Delta_{r}=\frac{\partial}{\partial z_{r}}+\Delta_{r+1}^{r} \frac{\partial}{\partial z_{r+1}}+\Delta_{\nu}^{r} \frac{\partial}{\partial z_{\nu}}, \\
& \Delta_{r+1}=\quad \Delta_{r+1}^{r+1} \frac{\partial}{\partial z_{r+1}}+\Delta_{\nu}^{r+1} \frac{\partial}{\partial z_{\nu}}, \\
& \Delta_{k}=\quad \Delta_{r+1}^{k} \frac{\partial}{\partial z_{r+1}}+\Delta_{\nu}^{k} \frac{\partial}{\partial z_{\nu}}, z_{j}=w_{j},
\end{aligned}
$$

where $\Delta_{j}^{i}(0)=0$ for every $i=r+1, \ldots, k$ and $j=r+1, \ldots, \nu$.

Indeed, taking the inverse matrix of $\left\|\Delta_{j}^{i}(0)\right\|$, i.e. $\left\|\Delta_{j}^{i}(0)\right\|^{-1}:=\left\|\delta_{j}^{i}\right\|$, we introduce the new vector fields

$$
\Delta_{j}^{\prime}=\sum_{j+1}^{r} \delta_{j}^{i} \Delta_{j}, \quad i=1, \ldots, r,
$$

as generators. After easy calculations, we obtain the required form for the generators.

Now, set

$$
f\left(z_{1}, \ldots, z_{r}, w_{r+1}, \ldots, w_{\nu}\right)=z_{j}-g_{j}\left(z_{1}, \ldots, z_{r}\right), \quad z_{j}=w_{j},
$$

for $j=r+1, \ldots, \nu$, where the $g_{j}$ are formal power series

$$
g_{j}(z)=\sum a_{\alpha} \zeta^{\alpha}, \quad z^{2}=\left(z_{1}^{2}, \ldots, z_{r}^{2}\right), \zeta=z^{2},
$$

$\alpha:=\left(\alpha_{1}, \ldots, \alpha_{r}, 0, \ldots, 0\right)$ and $a_{\alpha} \in \mathbb{C}$. Having in mind (4.5) we obtain

$$
\Delta_{i}\left(g_{j}\right)=\partial g_{j} / \partial z_{i} \quad \text { for } 1 \leq i \leq r .
$$

Then in view of the ordinary Taylor formula, we set

It follows that

$$
\frac{\partial^{\alpha_{1}+\ldots+\alpha_{r}} g}{\partial \zeta_{1}^{\alpha_{1}} \ldots \partial \zeta_{r}^{\alpha_{r}}}(0)=a_{\alpha} \alpha !|\alpha| ! \nu^{|\alpha|} .
$$

$$
\Delta^{\alpha}\left(g_{j}\right)(0)=a_{\alpha} \alpha !|\alpha| ! \nu^{|\alpha|} .
$$

On the other hand, $\Delta^{\alpha}\left(f_{j}\right)=\Delta^{\alpha}\left(z_{j}\right)-\Delta^{\alpha}\left(g_{j}\right)$. Thus by (4.1) the ideal $\left(f_{r+1}, \ldots, f_{\nu}\right)$ is $\mathcal{D}$-stable only if $\Delta^{\alpha}\left(z_{j}\right)(0)-\Delta^{\alpha}\left(g_{j}\right)(0)=0$ for each $\alpha$. In 
view of $(4.6) g_{j}(z)$ obtains the form

$$
g_{j}(z)=\sum_{\alpha} \frac{\Delta^{\alpha}\left(g_{j}\right)(0)}{|\alpha| ! \alpha ! \nu^{|\alpha|}} \zeta^{\alpha}=\sum_{\alpha} \frac{\Delta^{\alpha}\left(z_{j}\right)(0)}{|\alpha| ! \alpha ! \nu^{|\alpha|}} z^{2 \alpha} .
$$

The local convergence follows from (3.8).

For the second statement we consider the product

$$
\mathbb{C}^{\nu-r}\left(w_{r+1}, \ldots, w_{\nu}\right) \times U,
$$

where $U$ is an open neighborhood in $\mathbb{C}^{r}\left(z_{1}, \ldots, z_{r}\right)$ on which the holomorphic functions $g_{j}$ are defined. Denote by $Z$ the closed complex subspace of the above product, defined by the ideals generated by $f_{j}$. Then $\operatorname{rank}_{x}\left(f_{r+1}, \ldots, f_{\nu}\right)=\nu-r$ and $\operatorname{dim}_{x} Z=r$ for all $x \in Z$. The functions $f_{r+1}, \ldots, f_{\nu}$ generate all ideals of $Z$, i.e. all $J(Z)_{x}$ for $x \in Z$, as every analytic set $A$ is in a canonical way a closed complex subspace with structure sheaf $\left(\mathcal{O}_{Z} / J(A)\right) \mid A$. By (4.2) we get $\operatorname{emb}_{x} Z+\nu-r=\nu-r+r$ for all $x \in Z$. Hence $\operatorname{emb}_{x} Z=r=\operatorname{dim}_{x} Z$ for all $x \in Z$. By (4.3) the statement is proved.

5. Local holonomicity. Having the differential system $(M, \mathcal{D})$ take the sequence of subsheaves of $\mathcal{T}_{M}$

$$
\mathcal{D}=\mathcal{D}_{1} \subset \mathcal{D}_{2} \subset \ldots \subset \mathcal{D}_{h(K)}=\ldots
$$

To each system $\mathcal{D}_{j}$ we assign the ideal of all germs $f$ at the points $p$ such that $\Delta^{\alpha}(f)(p)=0$, where $\Delta^{\alpha}$ is constructed from $\mathcal{D}_{j}$. We get $J_{\mathcal{D}_{1}} \supset J_{\mathcal{D}_{2}} \supset$ $\ldots \supset J_{\mathcal{D}_{h(K)}}=\ldots$

Proposition 5.1. If an ideal $I$ is $\mathcal{D}$-stable, then it is also $\mathcal{D}_{j}$-stable, $j=1, \ldots, h(K)$.

P r o of. If $\Delta, \Delta^{\prime} \in \mathcal{D}$, we have $\Delta(I) \subset I$ and $\Delta^{\prime}(I) \subset I$, which implies $\left(\Delta \circ \Delta^{\prime}-\Delta^{\prime} \circ \Delta\right)(I) \subset I$. So we obtain $\Delta^{\prime \prime}(I) \subset I$ for every $\Delta^{\prime \prime} \in \mathcal{D}_{2}$, etc.

Proposition 5.2. The ideal $J_{\mathcal{D}_{h(K)}}$ is $\mathcal{D}_{h(K)}$-stable.

$\mathrm{Proof}$. It is not difficult to see that

$$
\Delta^{\alpha}\left(\Delta_{i}(f)\right)=\Delta^{\alpha+\gamma}(f)+P \Delta(f),
$$

where $\gamma=(0, \ldots, 1, \ldots, 0)(1$ is in the $i$ th position $)$ and $P \Delta$ is a polynomial of $\Delta_{1}, \ldots, \Delta_{k}$ of degree less than $|\alpha+\gamma|$. The above equality is true because $\mathcal{D}_{h(K)}$ is a Lie algebra.

Now let $\widetilde{\Delta}_{1}, \ldots, \widetilde{\Delta}_{k}$ be a base of $\mathcal{D}_{h(K)}$. It is enough to show that $\widetilde{\Delta}_{i}(f) \in J_{\mathcal{D}_{h(K)}}, i=1, \ldots, k$, for every $f \in J_{\mathcal{D}_{h(K)}}$. But this follows by induction, on the length of the multi-index $\alpha$, from the equality

$$
\Delta^{\alpha}\left(\widetilde{\Delta}_{i}(f)\right)(p)=\Delta^{\alpha+\gamma}(f)(p)+P \Delta(f)(p),
$$


since $\Delta^{\alpha+\gamma}(f)(p)=P \Delta(f)(p)=0$.

From Propositions 5.1 and 4.1 we conclude that $\left(f_{r+1}, \ldots, f_{\nu}\right) \subset J_{\mathcal{D}_{h(K)}}$. Using the Weierstrass division theorem we can also prove the inverse inclusion. Indeed, if $f \in J_{\mathcal{D}_{h(K)}}$ we divide it by $f_{r+1}$. Since $f_{r+1}$ is of order 1 in $z_{r+1}$ we get $f=Q_{r+1} f_{r+1}+R_{r+1}$, where the remainder $R_{r+1}$ does not depend on $z_{r+1}$. Dividing $R_{r+1}$ by $f_{r+2}$ and so on, we get finally $f=Q_{r+1} f_{r+1}+Q_{r+2} f_{r+2}+\ldots+Q_{\nu} f_{\nu}+R_{\nu}$, where $R_{\nu}$ is 0 .

Recapitulating, we find that $\left(f_{r+1}, \ldots, f_{\nu}\right)$ is a $\mathcal{D}_{h(K)}$-stable ideal.

Remark. In general, the obtained result is of local character. It is interesting to construct a maximal integral subspace.

Examples 5.3. 1) Consider $\left(\mathbb{C}^{3}, \Delta\right)$, where $\Delta=\partial / \partial z_{2}+z_{1} \partial / \partial z_{3}$. This is a holonomic holomorphic differential system whose singular integral is the closed subspace defined by $z_{3}-z_{1} z_{2}=0$.

2) Now we take the holomorphic differential system $\left(\mathbb{C}^{3}, \mathcal{D}\right)$ where $\mathcal{D}$ is defined globally by the vector fields $\Delta_{1}=\partial / \partial z_{1}$ and $\Delta_{2}=\partial / \partial z_{2}+$ $z_{1} z_{3} \partial / \partial z_{3}$. It is easy to calculate that $\left[\Delta_{1}, \Delta_{2}\right]=z_{3} \partial / \partial z_{3}$ and, following the method of 4.4 , that $\Delta^{\alpha}\left(g_{3}\right)=z_{3} \Delta^{\alpha}\left(z_{1}\right)$. So, we see that on every compact $K$ in the vector subspace defined by $z_{3}=0$, the series $g_{3}$ is zero and $f_{3}=z_{3}-g_{3}$ is even zero on the whole subspace $z_{3}=0$. This means that $h(K)=h\left(z_{3}=0\right)=1$, or that the maximal integral subspace is the complex manifold defined by $z_{3}=0$.

3) The system $\left(\mathbb{C}^{3}, \Delta_{1}=\partial / \partial z_{1}, \Delta_{2}=\partial / \partial z_{2}+z_{1} \partial / \partial z_{3}\right)$ is not holonomic as $\left[\Delta_{1}, \Delta_{2}\right]=\partial / \partial z_{3}$. The completed system $\mathcal{D}_{1}=\left\{\Delta_{1}, \Delta_{2}, \partial / \partial z_{3}\right\}$ defines a Lie algebra sheaf, i.e. the index of non-holonomicity is 1 .

\section{References}

[1] S. Fischer, Complex Analytic Geometry, Lecture Notes in Math. 538, Springer, 1976.

[2] H. Grauert and R. Remmert, Coherent Analytic Sheaves, Springer, 1984.

[3] A. M. Vershik and V. Ya. Gershkovich, Nonholonomic dynamical systems. Geometry of distributions and variational problems, in: Sovrem. Probl. Mat. Fund. Napravl. 16, VINITI, Moscow 1987, 5-85 (in Russian).

INSTITUTE OF MATHEMATICS

BULGARIAN ACADEMY OF SCIENCES

P.O. BOX 373

1090 SOFIA, BULGARIA

Reçu par la Rédaction le 12.9.1990 